\title{
Environmental dependencies in the luminosity function of galaxies
}

\author{
R. Brent Tully \\ Institute for Astronomy, University of Hawaii, Honolulu, HI 96822, USA \\ email: tully@ifa.hawaii.edu
}

\begin{abstract}
The evidence is becoming strong that the luminosity function of galaxies varies with environment. Higher density, more dynamically evolved regions appear to have more dwarfs per giant. The situation is becoming clearer as a result of wide field imaging surveys with the Canada-France-Hawaii and Subaru telescopes and spectroscopy of faint dwarfs with the Keck telescope. We report here on extensive observations of the small but dense NGC 5846 Group. The faint end of the luminosity function rises relatively steeply in this case.
\end{abstract}

Keywords. galaxies: luminosity function, galaxies: dwarf

\section{Introduction}

At the inception of this program, circa 1997, the following thoughts provided motivation. The properties of the luminosity function of galaxies at the time were poorly constrained by observations at the faint end. Most of the observational attention had been directed toward rich clusters of galaxies because they could provide good statistics and contrast against interlopers. However rich clusters are relatively distant and at faint levels the luminosity function is only defined after statistical corrections for contamination. Experience in and near the Local Group teaches us that the vast majority of dwarf galaxies are low surface brightness, a property that distinguishes them from background objects. If we confine a study to groups that are clean from the perspective of confusion from nearby structures then we might reliably identify group members without need for statistical corrections. By looking at nearby groups, we can observe down to faint magnitudes, over a variety of environments including some where the density is low.

We began with a study of the Ursa Major (UMa) Cluster because we were familiar with the region from work on the bright members (Tully et al. 1996; Verheijen \& Sancisi 2001). The UMa Cluster almost qualifies as Abell richness class 0 but it is different from most clusters to receive attention because it is irregular, low density, and spiral rich. The cluster is at roughly the same distance as the Virgo Cluster and subtends $100 \mathrm{sq}$. deg. We sampled $15 \%$ of the area with strips along the major and minor axes of the elongated cluster with observations using the $12 \mathrm{k}$ imager at the Canada-France-Hawaii Telescope and the neutral hydrogen receiver at the Very Large Array. We conducted a double blind search for dwarf galaxies with Neil Trentham conducting the optical search and Marc Verheijen conducting the HI search.

The HI survey turned up all the previously known galaxies including a couple that had excaped detection in HI but only uncovered 11 new objects. The HI mass flunction in UMa was demonstrated to be flat at low masses (Verheijen et al. 2000). The optical survey turned up 3 times as many candidates, still not a large number. Probably many, if not most, of these candidates would have been detected in HI if the sensitivity achieved with the VLA could have been pushed a factor 10 below our $10^{7} M_{\odot}$ limit. In any event, 


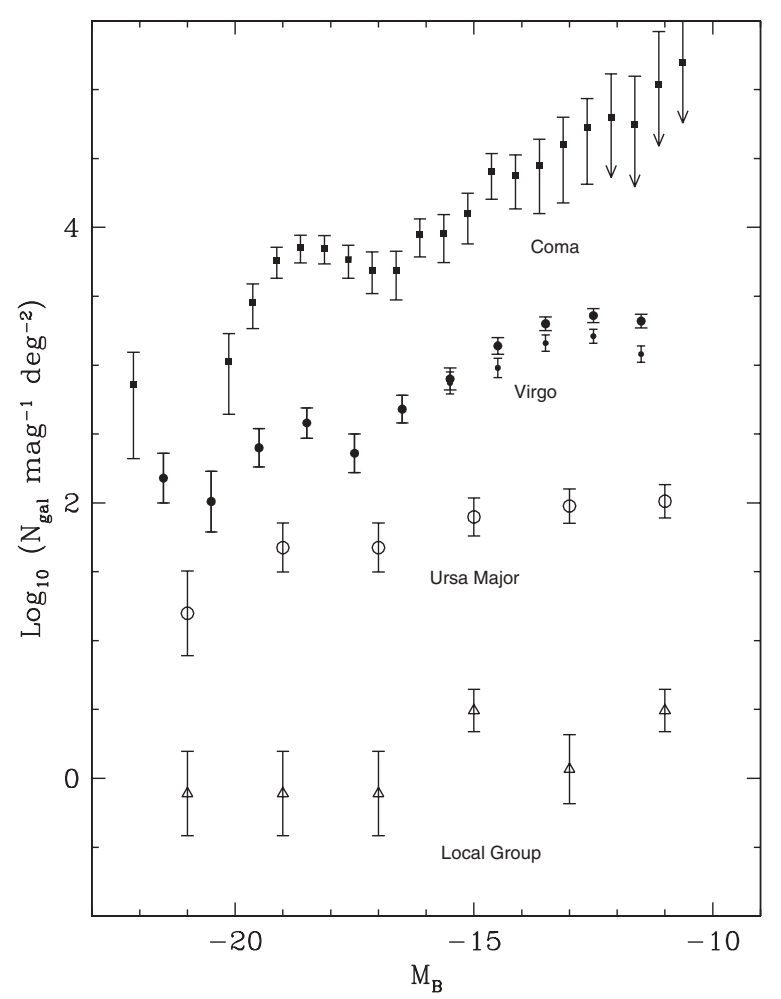

Figure 1. B-band luminosity functions for the Coma, Virgo, Ursa Major clusters and the Local Group. Vertical scales are shifted for clarity. Downward arrows in the case of the Coma Cluster reflect background contamination uncertainties.

the optical luminosity function in UMa is almost flat at the faint end (see Figure 1 extracted from Trentham et al. 2001).

\section{Environmental Dependencies?}

The luminosity function in the UMa Cluster is flatter than others have found in denser, more dynamically evolved places, reminiscent of the situation found in the Local Group (Klypin et al. 1999; Moore et al. 1999). The luminosity function in these low density environments is much flatter than the mass function anticipated by $\Lambda C D M$ hierarchical clustering theory (Sheth \& Tormen 1999). The theory predicts that the mass spectrum is steeper in lower density environments, not flatter (Sigad et al. 2000). What could explain an increased departure of the luminosity function from the mass function in lower density environments?

Thoul \& Weinberg (1996), Gnedin (2000) and others have discussed the way reionization could have suppressed the accumulation of gas in small dark matter halos. Hot intergalactic gas has too much energy to fall into a small dark matter halo that collapses after the epoch of reionization. By contrast, a small halo that collapses before reionization will be accompanied by cold gas. Early forming low mass halos would contain gas and form stars while late forming low mass halos would not contain gas, hence not be the sites of star formation. This scenario could explain the trends seen in the properties 


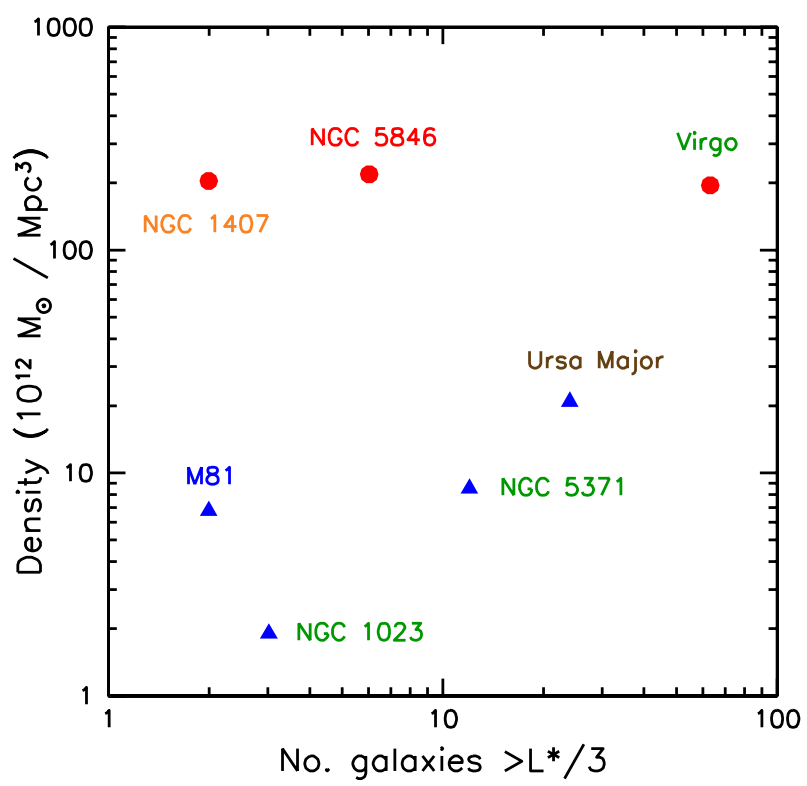

Figure 2. Seven cluster or group environments will be surveyed over the course of this program. The environments are characterized by a density parameter (virial mass / volume) and a richness parameter (number of members more luminous than $L^{\star} / 3$ ). Groups of predominantly early-type and late-type galaxies are identified by circles and triangles, respectively. The present paper summarizes results obtained for the dense but relatively poor NGC 5846 Group.

of the luminosity function because low mass halos collapse earlier in environments that become rich clusters than in low density environments like those that become the Local Group or the Ursa Major Cluster (Tully et al. 2002). Semi-analytic models suggest that gas retention is strongly favored in places that become dense, massive clusters because some halos form very early. Even in these densest places, though, most halos will form after reionization; ie, gas will not be pulled in with the collapse of most halos.

\section{Pilot Program to Survey a Variety of Environments}

Our next step was to take a quick look at a wide variety of environments within the Local Supercluster. Subaru SuprimeCam was used to image the inner parts of 5 additional groups, which added to UMa gave us 6 samples (Trentham \& Tully 2002). Trends were seen in the suspected sense: more dynamically evolved regions have higher dwarf/giant ratios. The mean slope at the faint end of the luminosity function is characterized by a Schechter (1976) slope parameter $\alpha=-1.19 \pm 0.03$. However, not enough sky was surveyed in each group for sufficient statistics on individual groups.

The problem required much more sky coverage. Entire groups should be surveyed and groups with very different properties should be considered. We began a program with the wide field imagers on the Canada-France-Hawaii Telescope, culminating in the use of the 1 sq. deg. field MegaCam. Fields were chosen to maximize the range of group properties from numerically rich to poor in bright galaxies and from dense and dynamically evolved to sparse and dynamically young. The seven groups identified in Figure 2 have been or will be observed. We report here on the case of the NGC 5846 Group. 


\section{The Small, Dense NGC 5846 Group}

The number of luminous galaxies in the NGC 5846 Group is modest, with only $3 L^{\star}$ galaxies, but the group is dynamically evolved, with almost all members of early type. It is a dumbbell system: members congregate around the two bright ellipticals NGC 5813 and NGC 5846. Extended X-ray emission is centered on these two major galaxies. The distance to the group is $26 \mathrm{Mpc}$. The velocity dispersion, from 86 redshifts, is $322 \mathrm{~km} / \mathrm{s}$, resulting in a virial mass estimate of $8 \times 10^{13} M_{\odot}$ and mass to light ratio of 320 in solar units.

The definition of the luminosity function to faint levels depends on the ability to identify group members free of contamination. We first select candidates based on quantitative surface brightness criteria that would assure inclusion of dwarfs seen in the local neighborhood and redshift-confirmed members of the UMa and Virgo clusters. These low surface brightness candidates were then evaluated on morphological grounds and given qualitative group membership ratings (1) probable, (2) possible, (3) plausible, and (4) implausible.

Our rating scheme was then tested in two ways. The first and most rigorous test was to obtain new velocities. The Sloan Digital Sky Survey (SDSS) now provides redshifts for essentially all our galaxies with $R<17\left(M_{R}<-15\right)$. We have obtained additional spectra for galaxies up to 2 mag fainter with Keck Telescope. It was found that (a) all 24 galaxies with new velocities that were rated 1 and 2 are members, (b) 16 of 23 galaxies rated 3 are members, (c) only 1 galaxy rated 4 has a new velocity that shows it to be a member, and (d) 5 high surface brightness galaxies have turned out to be members. The second test involves intercomparisons of spatial correlations. Galaxies rated 1 and 2 are as highly correlated with the group as galaxies confirmed as members on the basis of velocities, indicating that essentially $100 \%$ of these objects belong to the group. By contrast, the correlation with the galaxies rated 3 indicates that $50 \% \pm 10 \%$ of these candidates are in the group. It is concluded that $255 \pm 15$ of our candidates are associated with the group. Of the dwarfs, $80 \%$ are early types.

There turns out to be a clean separation between high and low surface brightness objects in plots of surface brightness vs. luminosity. There are 3 very compact ellipticals; one rather like M32. It turns out that these three unusual objects are the nearest neighbors in projection to the dominant NGC 5846 galaxy. We suggest that these 3 galaxies have been tidally truncated.

The identification of group members allows us to build the luminosity function seen in Figure 3. There is a saddle point in the observed distribution at $M_{R} \sim-20$, similar to a feature seen in other samples, and not something that can be described by a Schechter function. Following our procedure in the Subaru pilot program (Trentham \& Tully 2002), we fit the luminous, high surface brightness galaxies with a Gaussian function $\left(M_{g}=-21.0, \sigma_{g}=1.1^{m}\right)$ and fit the faint, low surface brightness component with a Schechter function $\left(M_{d}^{\star}=-19.5, \alpha_{d}=-1.36\right)$. The luminosity function is well defined to a completion limit of $M_{R}=-12$.

The luminosity function for the NGC 5846 Group is compared with what was found from other environments in Figure 4. The faint end slope of $\alpha=-1.36 \pm 0.04$ is significantly steeper than the mean slope for the 5 Subaru groups of $\alpha=-1.19 \pm 0.03$, and steeper still than the slope $\alpha \sim-1.0$ found for the UMa sample. However it is shallower than the Press-Schechter mass function slope. The NGC 5846 Group environment is dense and dynamically evolved, even though the number of bright galaxies is modest and the total mass is not extreme. Our information is still limited but we are seeing a 


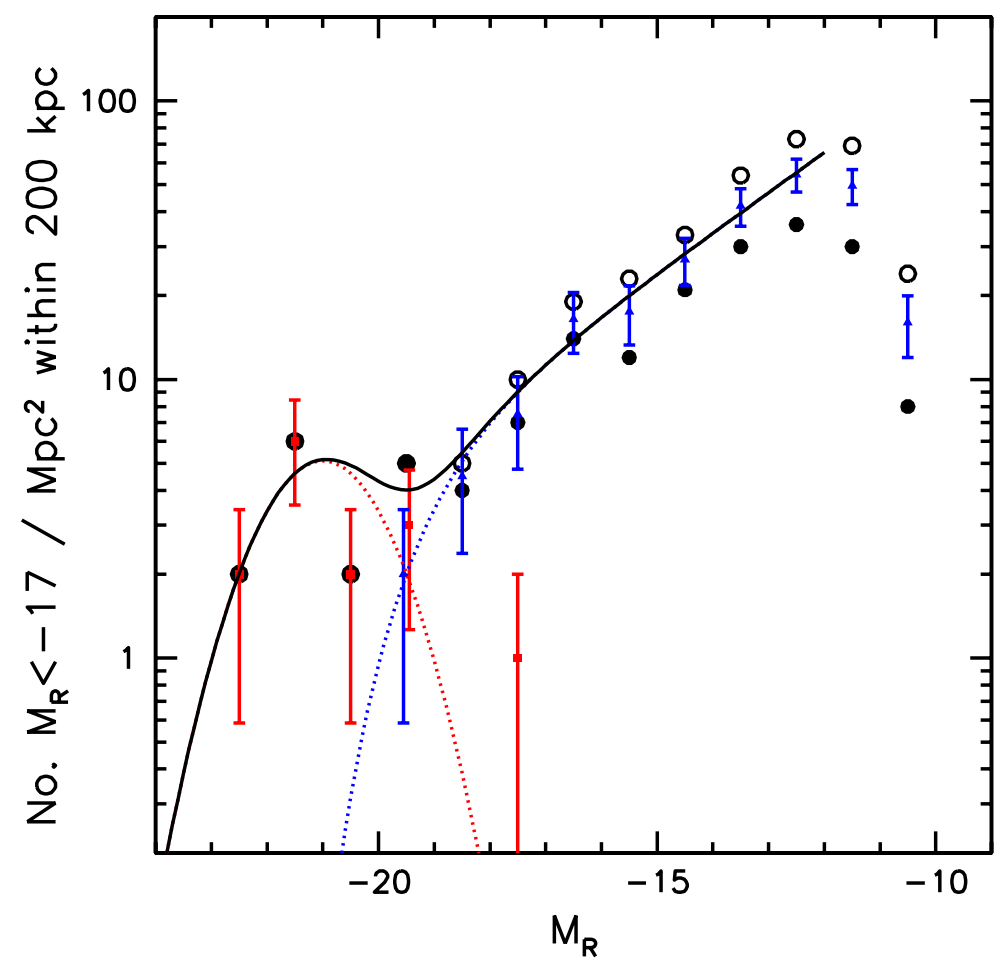

Figure 3. NGC 5846 Group luminosity function normalized by the surface density of galaxies with $M_{R}<-17$ at $200 \mathrm{kpc}$ radius. Large filled circles: number of galaxies per half-magnitude bin either confirmed as a group member with a redshift or almost a certain member with a morphological rating 1 or 2 . Large open circles: same except also including morphological rating 3. Small filled squares: high surface brightness only. Small filled triangles: low surface brightness only, splitting the difference between the values represented by the filled and open large circles. Dotted curves: separate fits to the high and low surface brightness distributions. Solid curve: sum of the two component fits. Parameters of the fit: $M_{g}=-21.0 \pm 0.3, \sigma_{g}=1.1 \pm 0.2, N_{g}=5.1 \pm 1.2$, $M_{d}=-19.5 \pm 0.6, \alpha_{d}=1.36 \pm 0.04, N_{d}=5.9 \pm 0.4$.

pattern that appears consistent with the reionization model (Tully et al. 2002). Details of the NGC 5846 study are presented by Mahdavi et al. (2005).

\section{Acknowledgements}

My collaborators in this work are Neil Trentham, Andisheh Mahdavi, Rachel Somerville, and Marc Verheijen. Support is provided by the US National Science Foundation award AST-03-07706.

\section{References}

Gnedin, N.Y. 2000, ApJ 542, 535

Klypin, A., Kratsov, A.V., Valenzuela, O. \& Prada, F. 1999, ApJ 522, 82

Mahdavi, A., Trentham, N. \& Tully, R.B. 2005, AJ to be submitted

Moore, B., Ghigna, S., Governato, F., Lake, G., Quinn, T., Stadel, J. \& Tozzi, P. 1999, ApJ 524, L19

Schechter, P.L. 1976, ApJ 203, 297

Sheth, R.K. \& Tormen, G. 1999, MNRAS 308, 119 


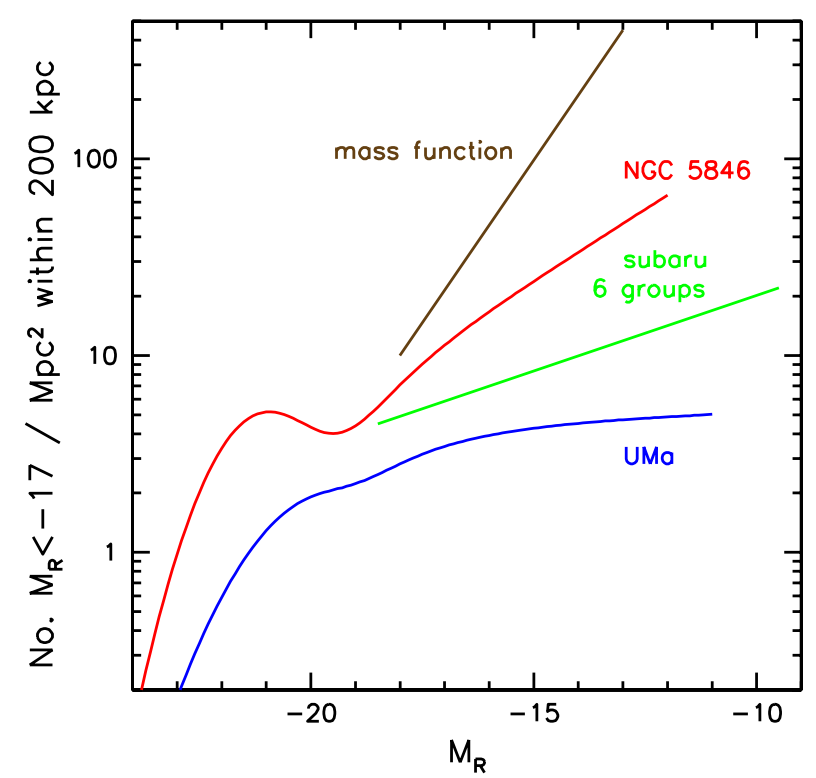

Figure 4. Schematic comparison of the NGC 5846 Group luminosity function with other cases. The curve representing the NGC 5846 Group is the solid curve in Fig. 3. The curves labeled 'subaru 6 groups' and 'UMa' are taken from Trentham \& Tully 2002. The 6 groups curve represents an average found for the dwarf regime in 6 groups discussed by Trentham \& Tully 2002. The density normalizations are carried out in a consistent way in these separate cases. The steeper line labeled 'mass function' indicated the low mass slope of a modified Press-Schechter distribution with arbitrary vertical scaling.

Sigad, Y. et al. 2000, astro-ph/0010222

Thoul, A.A. \& Weinberg, D. 1996, ApJ 465, 608

Trentham, N. \& Tully, R.B. 2002 MNRAS 335, 712

Trentham, N., Tully, R.B. \& Verheijen, M.A.W. 2001 MNRAS 325, 385

Tully, R.B., Somerville, R.S., Trentham, N. \& Verheijen, M.A.W. 2002, ApJ 569, 573

Tully, R.B., Verheijen, M.A.W., Pierce, M.J., Huang, J.S., \& Wainscoat, R.J. 1996, AJ 112, 2471

Verheijen, M.A.W. \& Sancisi, R. 2001, A\&A 370, 765

Verheijen, M.A.W., Trentham, N., Tully, R.B. \& Zwaan, M.A. 2000 2000, ASP Conf. Ser. 218, 263

\section{Discussion}

GALLAGHER: (Great project!) Have you found a way to combine your model associating cluster dwarfs with primordial fluctuations with the observations of intermediate ages (velocity distributions, stellar pop ages) in cluster $\mathrm{dE} / \mathrm{dSph}$ galaxies?

TULLY: Archaic populations are found in almost all dwarfs where an appropriate observation has been made. As you say, some $\mathrm{dE} / \mathrm{dSph}$ manifest intermediate age populations as well. Our model suggests why some dwarf halos would start out with gas and have the capability of forming stars. It says nothing concerning the rate of conversion of this gas into stars. 